\title{
Continuing Umbrella of Research Experience for Underserved Minorities (CURE) Program
}

National Cancer Institute

\section{Source}

National Cancer Institute. Continuing Umbrella of Research Experience for Underserved

Minorities (CURE) Program. NCI Thesaurus. Code C19732.

The Continuing Umbrella of Research Experience for Underrepresented Minorities

Program (CURE) is a new strategy being implemented by the National Cancer Institute to expose minorities to cancer research at the high school and undergraduate levels. The program is being initiated nationwide. 